\title{
PERFIL DOS ÉSTERES OBTIDOS PELA TRANSESTERIFICAÇÃO ETANÓLICA E METANÓLICA DO ÓLEO DE NABO FORRAGEIRO
}

\author{
M. PAGNONCELLI ${ }^{1}$, D. TOSS ${ }^{1}$, M. GODINHO ${ }^{1}$ \\ ${ }^{1}$ Universidade de Caxias do Sul, Departamento de Ciências Exatas e Tecnologia, \\ e-mail: marlovap@yahoo.com.br
}

\begin{abstract}
RESUMO - Atualmente é indiscutível a busca por combustíveis renováveis, que visam substituir os de origem fóssil. Desta forma, o presente trabalho tem como objetivo avaliar o perfil de ésteres da transesterificação via catálise homogênea (hidróxido de sódio) pelas rotas metílica e etílica. Para a produção do biodiesel foram utilizados ácidos graxos de nabo forrageiro. A oleaginosa apresenta importante potencial para a produção de biocombustível por apresentar grande quantidade de óleo. Para os experimentos, utilizou-se a prensagem, obtendo-se 15,80\% de óleo. As reações de transesterificação percorreram com uma razão molar álcool:éster de 6:1, $1 \%$ de catalisador, tempo de $2,5 \mathrm{~h}$ e temperatura de $30^{\circ} \mathrm{C}$, para a reação com etanol e $65^{\circ} \mathrm{C}$ com metanol. O biodiesel obtido foi avaliado quanto ao perfil de ésteres por cromatografia gasosa (CG), onde foram identificados, como majoritários, o alquil éster de ácido oleico (cerca de 35\%), seguido do ácido erúcico, linoleico, linolênico, gadoleico, entre outros.
\end{abstract}

\section{INTRODUÇÃO}

$\mathrm{O}$ aumento da industrialização e o elevado crescimento populacional proporcionaram aumento na demanda energética, e consequentemente aumento no consumo de petróleo. Tais fatores geraram elevação do custo do petróleo e diminuição das reservas de combustível, além da elevação da emissão de gases causadores do efeito estufa. Visando amenizar tais problemas, busca-se alternativas para substituir os derivados do petróleo por combustíveis renováveis, como o biodiesel (Banković-ilić et al., 2012). Biodiesel é a denominação empregada para definir combustíveis biodegradáveis derivados de fontes renováveis. A produção de biodiesel pode ser realizada pelo processo de transesterificação de óleos vegetais ou gorduras animais, na presença de álcool e catalisador. A reação provoca a quebra da molécula de triglicerídeo em alquil ésteres (biodiesel) e glicerol, conforme Figura 1 (Abuhabaya et al., 2013; Holanda, 2004).

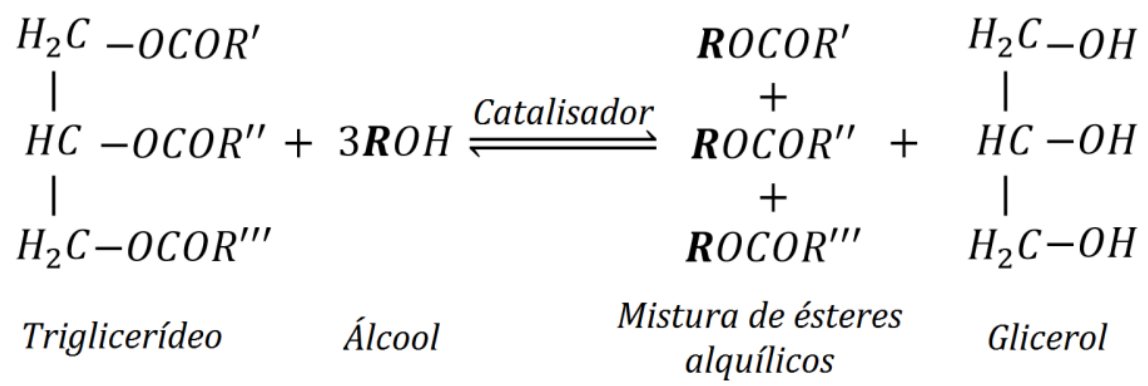

Figura 1 - Reação de transesterificação 
A soja é a oleaginosa mais utilizada para produção do biodiesel no Brasil, entretanto, em virtude dessa ser empregada para consumo humano vem-se buscando alternativas para substituí-la. O nabo forrageiro é uma oleaginosa que atende esse requisito (Valle et al.,2009). Este é uma planta de cultivo de inverno, utilizada como rotação de cultura por possuir elevada capacidade de reposição de nutrientes no solo, além disso, desenvolve-se bem em solos pobres de nutrientes e resiste a baixas temperaturas sem sofrer danos, podendo ser cultivada no sul, sudeste e centro oeste do Brasil (Barros e Jardine, 2013; Ávila e Sodré, 2012). A Figura 2 representa a planta e as sementes do nabo forrageiro. Segundo Cremonez et al. (2013), o ácido oleico (C18:1) é o ácido graxo predominante na constituição do óleo vegetal proveniente do nabo forrageiro.

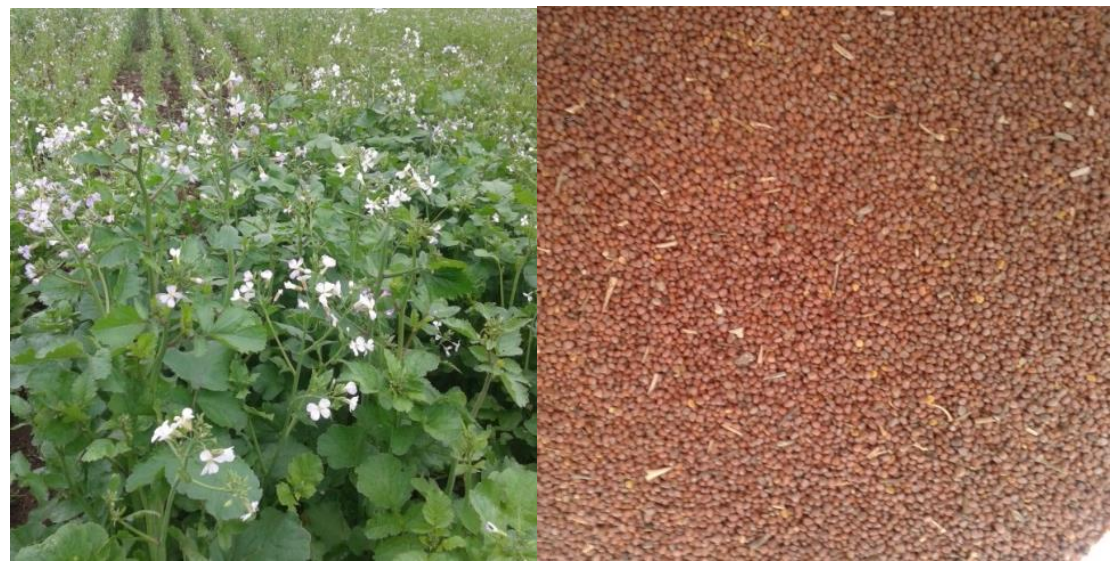

Figura 2 - Planta e sementes de nabo forrageiro

Segundo Banković-ilić et al. (2012), os ácidos graxos presentes no biodiesel estão diretamente relacionados a características físico químicas do biodiesel, como densidade, viscosidade, ponto de fulgor, ponto de névoa, estabilidade oxidativa índice de acidez, entre outros. Sendo assim o tipo de ácido graxo presente, dependendo do tamanho da cadeia e número e localização da instauração influencia em propriedades significativas para bom desempenho do biodiesel no motor. Sendo que matérias primas contendo altos níveis de ácidos graxos monoinsaturados são boas opções para a produção de biodiesel quando comparadas a matérias primas com altos níveis de ácidos graxos poli-insaturados (Chammoun et al., 2012).

O presente trabalho tem por objetivo determinar e quantificar os álquis ésteres de ácidos graxos presentes no o biodiesel produzido por transesterificação metílica e etílica de óleo de nabo forrageiro, mediante à análise por cromatografia gasosa.

\section{MATERIAIS E MÉTODOS}

\subsection{Obtenção do óleo de nabo forrageiro}

O nabo forrageiro utilizado foi adquirido da Agroindústria e Comércio Pozza Ltda, pertencente ao lote 06 da safra de 2012. Realizou-se a extração do óleo em uma mini prensa contínua, elétrica, com rosca sem fim, da marca Ecirtec, modelo MPE-40. O rendimento percentual de óleo foi calculado frente à massa úmida de sementes. 


\subsection{Reação de transesterificação}

Equipamento: As reações foram conduzidas num balão de vidro sobreposto por um condensador e aquecido por uma chapa de aquecimento com agitador magnético. O controle da temperatura foi realizado mediante a inserção de um termômetro no meio reacional. $\mathrm{O}$ equipamento experimental está ilustrado na Figura 3.

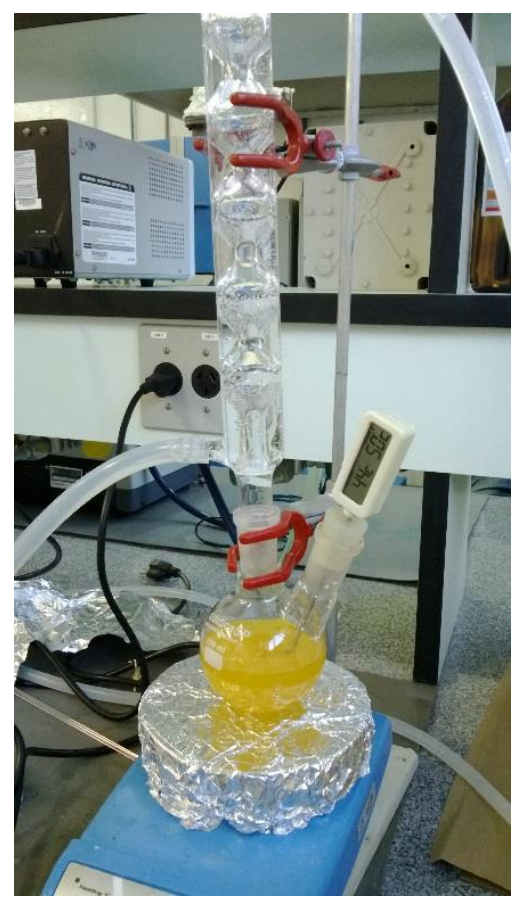

Figura 3 - Equipamento utilizado nas reações de transesterificação

Procedimento experimental: A reação foi realizada usando a proporção álcool/éster de 6:1 em mol e $1 \%$ de catalisador, sendo a massa molar do óleo de nabo forrageiro calculada mediante ao seu principal componente, o ácido oleico, de fórmula molecular $\mathrm{C}_{18} \mathrm{H}_{34} \mathrm{O}_{2}$. $\mathrm{O}$ tempo de reação foi fixado em 2,5h.

Para a reação com etanol utilizou-se $100 \mathrm{~g}$ de óleo de nabo forrageiro e $30,15 \mathrm{~g}$ de álcool etílico P.A., caracterizando a relação molar 6:1. Desta forma a massa de hidróxido de sódio foi 1,3g. O meio reacional foi mantido a $30^{\circ} \mathrm{C}$, seguindo o trabalho de Ávila e Sodré (2012). Para reação com metanol, seguindo a mesma razão molar, utilizou-se $100 \mathrm{~g}$ de óleo de nabo forrageiro e $20,97 \mathrm{~g}$ de álcool metílico P.A. Desta forma $1,21 \mathrm{~g}$ de hidróxido de sódio foi utilizado como catalisador. $\mathrm{O}$ meio reacional foi mantido a $65^{\circ} \mathrm{C}$, seguindo o trabalho de Abuhabaya et al. (2013).

Após a reação, realizou-se a separação das fases glicerina (fase pesada) e biodiesel (fase leve), por diferença de densidade. Posteriormente o biodiesel foi neutralizado com ácido sulfúrico $0,5 \%$ e lavado com água até atingir $\mathrm{pH} 7$. Após a lavagem, as amostras foram centrifugadas, em centrífuga Novatécnica, modelo NT825, com rotação de 2200rpm, para remoção dos sabões e água. O fluxograma do procedimento experimental está ilustrado na Figura 4. 


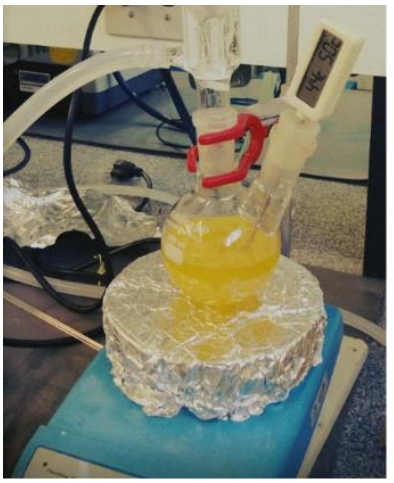

1 - Reação de transesterificação

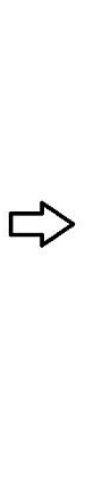

\section{2 - Separação das} fases

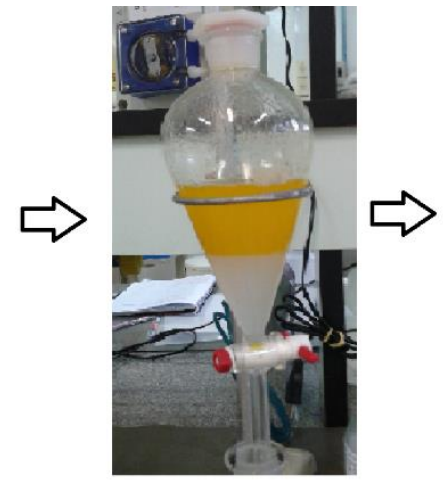

3 - Neutralização e lavagem

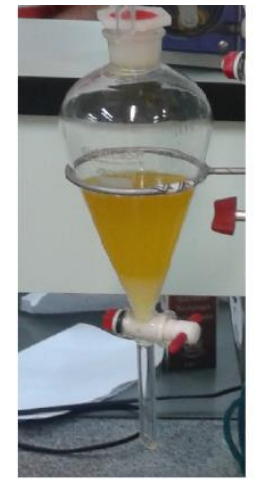

4 - Centrifugação

Figura 4 - Fluxograma do procedimento experimental

\subsection{Metodologia analítica}

As amostras de biodiesel foram analisadas num cromatógrafo gasoso (GC/MS) acoplado a detector seletivo de massas Hewlett Packard 6890/MSD5973, equipado com software HP Chemstation e espectroteca Wiley 275. Foi utilizada uma coluna capilar de sílica fundida HPInnowax (30 m x $250 \mathrm{~mm}$ ) 0,50 mm espessura de filme (Hewlett Packard, Palo Alto, USA). O programa de temperatura da coluna foi: $80^{\circ} \mathrm{C}(5 \mathrm{~min}), 230^{\circ} \mathrm{C}$ a $3^{\circ} \mathrm{C} / \mathrm{min}(30 \mathrm{~min})$; interface $310^{\circ} \mathrm{C}$; split ratio 1:25; gás de arraste $\mathrm{He}(40 \mathrm{~cm} / \mathrm{s})$; razão de fluxo: $1,0 \mathrm{~mL} / \mathrm{min}$; ; energia de ionização $70 \mathrm{eV}$; intervalo de aquisição de massas 45-550; volume injetado $1 \mathrm{~mL}$ diluído em hexano (1:10).

\section{RESULTADOS E DISCUSSÃO}

O processo de extração de óleo de nabo forrageiro permitiu obter 15,8\% (base úmida). Observa-se que este valor é inferior aos indicados na bibliografia, que, segundo Barros e Jardine (2013), o óleo pode estar presente numa concentração entre 27 a 42\%. Ressalta-se que no presente trabalho foi empregada a extração por prensagem e rendimentos mais elevados podem ser obtidos empregando-se outros métodos, como por exemplo, a extração por solvente.

A Figura 5 apresenta os cromatogramas do resultado da transesterificação do óleo de nabo forrageiro, obtidos por rota etílica e metílica. Na Tabela 1 encontram-se os alquis ésteres de ácidos graxos identificados pela cromatografia gasosa. 


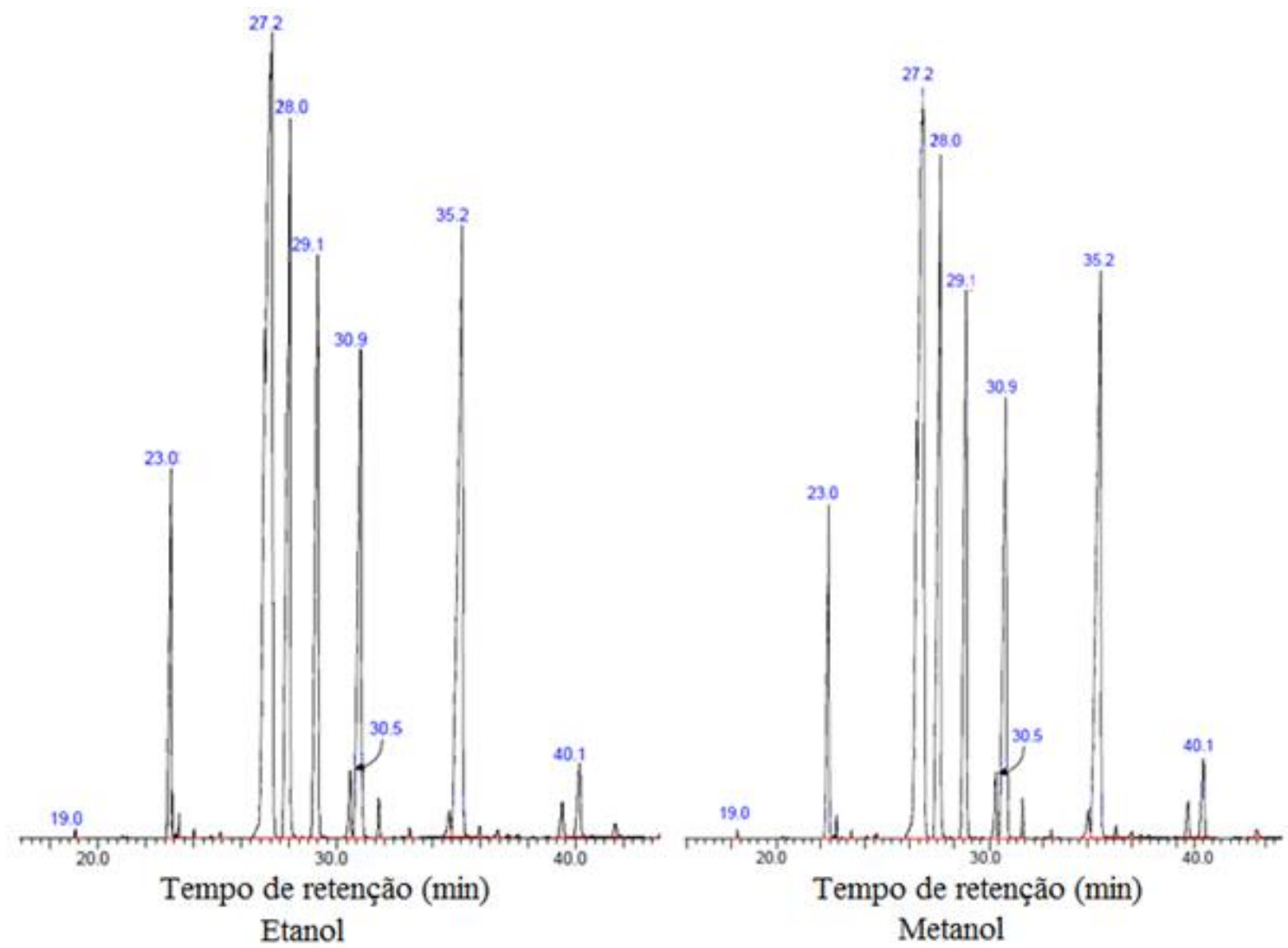

Figura 5 - Cromatografia gasosa do biodiesel via rotas etílica e metílica

Tabela 1 - Composição de alquis esteres do biodiesel de nabo forrageiro

\begin{tabular}{|c|c|c|c|c|c|}
\hline \multirow{2}{*}{$\begin{array}{l}\text { Tempo de } \\
\text { retenção } \\
(\min )\end{array}$} & \multirow[b]{2}{*}{ Composto } & \multirow{2}{*}{$\begin{array}{c}\text { Cadeia } \\
\text { carbônica }\end{array}$} & \multicolumn{3}{|c|}{ Quantidade (\% m/m) } \\
\hline & & & $\begin{array}{c}\text { Biodiesel } \\
\text { etanol }\end{array}$ & $\begin{array}{c}\text { Biodiesel } \\
\text { metanol }\end{array}$ & Bibliografia $^{1}$ \\
\hline 19,0 & Alquil éster de ácido mirístico & $\mathrm{C}_{14}$ & 0,07 & 0,07 & 6,0 \\
\hline 23,0 & Alquil éster de ácido palmítico & $\mathrm{C}_{16}$ & 5,13 & 5,01 & $7,0-8,3$ \\
\hline 27,2 & Alquil éster de ácido oleico & $\mathrm{C}_{18}$ & 35,49 & 34,87 & $29,1-34,5$ \\
\hline 28,0 & Alquil éster de ácido linoleico & $\mathrm{C}_{18}$ & 15,53 & 15,36 & $7,6-19,1$ \\
\hline 29,1 & Alquil éster de ácido linolênico & $\mathrm{C}_{18}$ & 11,15 & 11,11 & $4,6-13,2$ \\
\hline 30,5 & Alquil éster de ácido araquidico & $\mathrm{C}_{20}$ & 0,98 & 1,05 & $0,8-2,2$ \\
\hline 30,9 & Alquil éster de ácido gadoléico & $\mathrm{C}_{20}$ & 10,30 & 10,44 & $7,9-11,2$ \\
\hline 35,2 & Alquil éster de ácido erúcico & $\mathrm{C}_{22}$ & 17,43 & 18,05 & $11,9-33,3$ \\
\hline \multirow[t]{2}{*}{40,1} & Alquil éster de ácido nervônico & $\mathrm{C}_{24}$ & 1,43 & 1,56 & 2,0 \\
\hline & $\Sigma$ alquil ésteres & & 97,51 & 97,52 & \\
\hline
\end{tabular}

NOTA: ${ }^{1}$ Valle et al. (2009); Avila \& Sodré (2012).

Pela análise dos resultados da Tabela 1, observa-se semelhança entre a composição de alquil ésteres de ácidos graxos, obtidos via rota metílica e etílica, visto que estão relacionados com a 
composição do óleo vegetal de origem e não do processo de transesterificação. Para ambas as amostras aproximadamente $97,5 \%$ dos ácidos graxos foram identificados. Sendo que $6 \%$ desses são saturados (miristico e palmítico e araquídico), 65\% são monoinsaturados (oleico, erúcico, gadoléico e nervônico) e 26,5\% poli-insaturados (linoleico e linolênico).

Segundo Shah et al., (2013) o biodiesel proveniente da soja possui $24 \%$ de monoinsaturados e $60 \%$ de poli-insaturados. Ácidos graxos poli-insaturados tornam o biodiesel mais suscetível a oxidação, sendo assim o biodiesel proveniente do nabo possui estabilidade oxidativa superior a soja.

Quanto mais alto for o tamanho das cadeias do biodiesel e/ou o caráter saturado das moléculas, maior serão o ponto de névoa, ponto de entupimento de filtros de motores e ponto de fluidez do biodiesel. Tais aspectos devem ser levados em consideração pois influenciam na temperatura de utilização do combustível. O biodiesel de nabo, obtido no presente trabalho, possui em torno de $6 \%$ de compostos saturados e 67,4\% de compostos com cadeia de até 18 carbonos, o que o torna adequado para aplicação a baixas temperaturas (Lobo et al., 2009; Knothe et al., 2006).

A cromatografia gasosa confirmou a presenta de grande quantidade de ácido erúcico, aproximadamente 18\%. Segundo Wendlinger et al. (2014) este ácido graxo é tóxico. Assim, além de ser uma rotação de cultura, o nabo forrageiro não pode ser destinado à alimentação humana ou animal, como é observado para a soja e outras culturas de oleaginosas, contribuindo pela sua destinação na produção de biodiesel.

\section{CONCLUSÃO}

O ensaio de cromatografia gasosa pode ser considerado eficiente para determinação do perfil de álquil ésteres de ácidos graxos de biodiesel visto que os resultados obtidos permitem identificar e quantificar 97,5\% dos ácidos graxos presentes nas amostras de biodiesel.

Observou-se semelhança entre a composição de alquil ésteres de ácidos graxos, obtidos via rota metílica e etílica, pois estão diretamente relacionados à constituição da oleaginosa de origem. A presença de $65 \%$ de ácidos graxos monoinsaturados confere ao biodiesel, produzido com óleo de nabo forrageiro, maior estabilidade oxidativa quando comparado ao produzido com outras oleaginosas, como a soja, por exemplo, predominantemente constituída de ácidos graxos poliinsaturados, mais suscetíveis a oxidação.

\section{REFERÊNCIAS}

ABUHABAYA, A.; FIELDHOUSE, J.; BROWN, D. Influence of production variables for biodiesel synthesis on yields and fuel properties, and optimization of production conditions. Fuel, v. 103, n. 0, p. 963-969, 2013.

ÁVILA, R. N. D. A.; SODRÉ, J. R. Physical-chemical properties and thermal behavior of fodder radish crude oil and biodiesel. Industrial Crops and Products, v. 38, n. 0, p. 54-57, 2012.

BANKOVIĆ-ILIĆ, I. B.; STAMENKOVIĆ, O. S.; VELJKOVIĆ, V. B. Biodiesel production from non-edible plant oils. Renewable and Sustainable Energy Reviews, v. 16, n. 6, p. 3621-3647, 
2012.

BARROS, T. D.; JARDINE, J. G. Nabo forrageiro. 2013. Disponível em: <http://www.agencia.cnptia.embrapa.br/gestor/agroenergia/arvore/CONT000fbl23vn002wx5eo0sa wqe38tspejq.html>. Acesso em: 10 abril 2014.

CHAMMOUN, N.; GELlER, D. P.; DAS, K. C. Fuel properties, performance testing and economic feasibility of Raphanus sativus (oilseed radish) biodiesel. Industrial Crops and Products, v. 45, n. 0 , p. 155-159, 2013.

CREMONEZ, P. A.; FEIDEN, A.; CREMONEZ, F. E.; ROSSI, E.; ANTONELLI, J.; NEDALETI, W. C.; TOMASSONI, F. Nabo forrageiro: do cultivo a produção do biodiesel. Acta Iguazu, v. 2, n. 2, p. 64-72, 2013.

HOLANDA, A. Biodiesel e inclusão social. Brasília, DF. Câmara dos Deputados, Centro de Documentação e Informação, Coordenação de Publicações, 2004. 200 p. (Cadernos de altos estudos 1)

KNOTHE, G. et al. Manual de biodiesel. São Paulo: E. Blücher, 2006. 340 p.

LOBO, I. P.; FERREIRA, S. L. C.; CRUZ, R. S. Biodiesel: Parâmetros de qualidade e métodos analíticos. Quim. Nova, v. 32, n. 6, p. 1596-1608, 2009.

SHAH, S. N.; IHA, O. K.; ALVES, F. C. S.; SHARMA, B. K.; ERHAN, S. Z.; SUAREZ, P. A. Z. Potential application of Turnip Oil (Raphanus sativus L.) for biodiesel production: PhysicalChemical Properties of Neat Oil, Biofuels and their Blends with ultra-low sulphur diesel (ULSD). BioEnerg Reser, v. 6, n. 2, p. 841-850, 2013.

VALLE, P. W. P. A.; REZENDE, T. F.; SOUZA, R. A.; FORTES, I. C. P.; PASA, V. M. D. Combination of fractional factorial and Doehlert experimental designs in biodiesel production: Ethanolysis of Raphanus saivusI L. var. oleiferus Stokes oil catalyzed by sodium ethoxide. Energy \& Fuel, v. 23, p. 5219-5227, 2009.

WENDLINGER, C.; HAMMANN, S.; VETTER, W. Various concentrations of erucic acid in mustard oil and mustard. Food Chemistry, v. 153, n. 0, p. 393-397, 2014. 